\title{
Bacterial electrophysiology brought to light
}

An engineered fluorescent protein reveals electrical spikes in intact bacterial cells.

In 1854, Louis Pasteur, a visionary and pioneer in microbiology, said: "In the field of observation chance favors only the prepared mind." More than a century and a half later a rather serendipitous finding opens the door to a new realm in microbiological research.

Adam Cohen and his colleagues at Harvard University have been trying to develop a voltage-sensing protein for optogenetic studies of neuronal activity. They modified a light-driven proton pump of marine bacteria in a way that reverses the light-voltage coupling process: the engineered protein detects electrical fluctuations and translates them into optical signals. This proteorhodopsin optical proton sensor (PROPS) is an order of magnitude more sensitive than existing voltage-indicating proteins but efforts to express it in neuronal membranes have so far proven futile.
Part of these efforts involved recombinant expression in Escherichia coli. Out of curiosity, Cohen and colleagues looked at PROPSexpressing bacteria under a microscope and observed a notable phenomenon: bacterial cells exhibited cell-wide blinks in fluorescence consistent with electrical spiking, similar to that of firing neurons. Blinks typically lasted for a few seconds and were not synchronized among individual cells. They were abolished upon physical and chemical perturbations of aerobic respiration, indicating that the two processes were intrinsically coupled. The fluorescence blinks coincided with stepwise efflux of a cationic dye, suggesting that electrical spiking could have implications in bacterial stress response and continuous development of antibiotic resistance.

Although membrane potential has been long recognized as essential for bacterial survival, the small size of these organisms has prevented studies of individual cells. The layered structure of a bacterial envelope introduces additional complexity, making the membrane invisible to exogenous probes such as lipophilic voltage-sensitive dyes. PROPS is thus the first sensor to shed light on bacterial electrophysiology, and the authors are now trying to characterize the mechanism and purpose of electrical spiking in more detail. "All the proteins in the bacterial cell membrane share the same membrane potential," says Cohen. "By spiking back and forth, we think that the bacterium might be time-sharing its membrane potential between different values, each of which is optimal for a different kind of process."

\section{Petya V Krasteva}

\section{RESEARCH PAPERS}

Kralj, J.M. et al. Electrical spiking in Escherichia coli probed with a fluorescent voltage-indicating protein. Science 333, 345-348 (2011). 